\title{
Un nouveau pas dans I'utilisation du système Cre-LoxP chez les cellules souches embryonnaires de souris: la création de remaniements chromosomiques
}

Les lecteurs de médecine/sciences ont pu suivre les progrès de l'utilisation des cellules souches embryonnaires (cellules ES) de souris en vue d'introduire des modifications génétiques programmées chez l'animal [1-3]. Ces cellules, qui ont la capacité de coloniser la lignée germinale de la souris, peuvent au préalable être transfectées avec un $\mathrm{ADN}$ homologue d'un gène donné mais porteur d'une mutation; il en résulte le remplacement par recombinaison homologue $(\mathrm{RH})$ du gène en question par une version mutée. Les cellules ES ainsi modifiées permettent, via l'obtention de chimères germinales, la production de souris porteuses de la mutation. L'ensemble de cette stratégie a permis d'introduire des mutations nulles dans de très nombreux gènes et a eu un impact considérable notamment sur l'étude de la fonction des gènes $[4,5]$. De plus, dans une période récente, de nouveaux vecteurs de ciblage ont été élaborés, permettant de pallier certains inconvénients des vecteurs initialement employés, en particulier en tirant avantage des systèmes de recombinaison spécifique de site [6]. Il est ainsi devenu possible, même si la stratégie reste lourde à mettre en œuvre, d'une part, de créer des mutations "propres" (dépourvues de séquences de sélection qui pourraient interférer avec le fonctionnement du gène muté ou des gènes voisins) $[3,7,8]$, et, d'autre part, de contrôler dans le du gène muté, ce qui permet d'affiner son étude fonctionnelle $[3,8,9]$. L'ensemble de ces expériences concerne des modifications génétiques introduites dans un gène particulier.

Smith et al. viennent, dans un article récent [10], de franchir un pas supplémentaire en démontrant la possibilité, grâce à l'utilisation très élégante du système de recombinaison Cre-LoxP dans les cellules ES de souris, de créer des altérations à l'échelle chromosomique : ils ont ainsi obtenu des cellules ES porteuses d'une translocation réciproque entre les gènes $c$-myc sur le chromosome $15 \mathrm{et}$ les gènes d'immunoglobuline sur le chromosome 12.

Rappelons les principales propriétés du système de recombinaison Cre (pour causes recombination) - LoxP (pour locus of crossing-over). Ce système de recombinaison, découvert chez le bactériophage Pl et dont il a été montré qu'il pouvait fonctionner aussi bien chez les procaryotes que chez les eucaryotes, est relativement simple puisqu'il nécessite seulement l'activité d'une protéine, la recombinase, et la présence dans les molécules d'ADN que l'on veut recombiner de séquences particulières, les sites LoxP. La recombinase Cre reconnaît spécifiquement un site LoxP au sein duquel elle clive l'ADN puis catalyse la réunion de cet $\mathrm{ADN}$ avec une séquence d'ADN clivé à un autre site IoxP. Les sites LoxP, longs de 34 paires de bases ( $\mathrm{pb}$ ), sont constitués de deux séquences palindromiques de 13 pb séparées par une séquence centrale asymétrique de 8 pb (figure 1). C'est l'orientation relative de la séquence asymétrique centrale par rapport aux séquences qui la flanquent qui détermine le type de raboutage: par exemple, si deux sites LoxP sont placés dans une séquence d'ADN avec une orientation identique de la séquence centrale, l'action de la recombinase aboutit à la délétion du fragment d'ADN situé entre les deux sites: à l'inverse, si les séquences centrales sont d'orientations opposées, la recombinase entraîne l'inversion du fragment d'ADN. La recombinase Cre est également capable, bien qu'avec une moindre efficacité, d'induire la recombinaison entre deux molécules d'ADN différentes, chacune porteuse d'un site LoxP.

C'est cette dernière propriété du système Cre-LoxP qu'ont utilisé Smith $e t$ al. [10] pour créer une translocation réciproque entre les loci c-myc et $\lg H$ (chaînes lourdes des immunoglobulines), respectivement sur les chromosomes 12 et 15 , un type de translocation fréquemment rencontré dans les plasmocytomes de souris.

La stratégie employée comporte deux temps (figure 2): d'abord l'introduction par recombinaison homologue des sites LoxP dans une orientation appropriée par rapport aux centromères, respectivement dans les chromosomes 12 et 15 , ensuite l'expression de la recombinase de manière à 


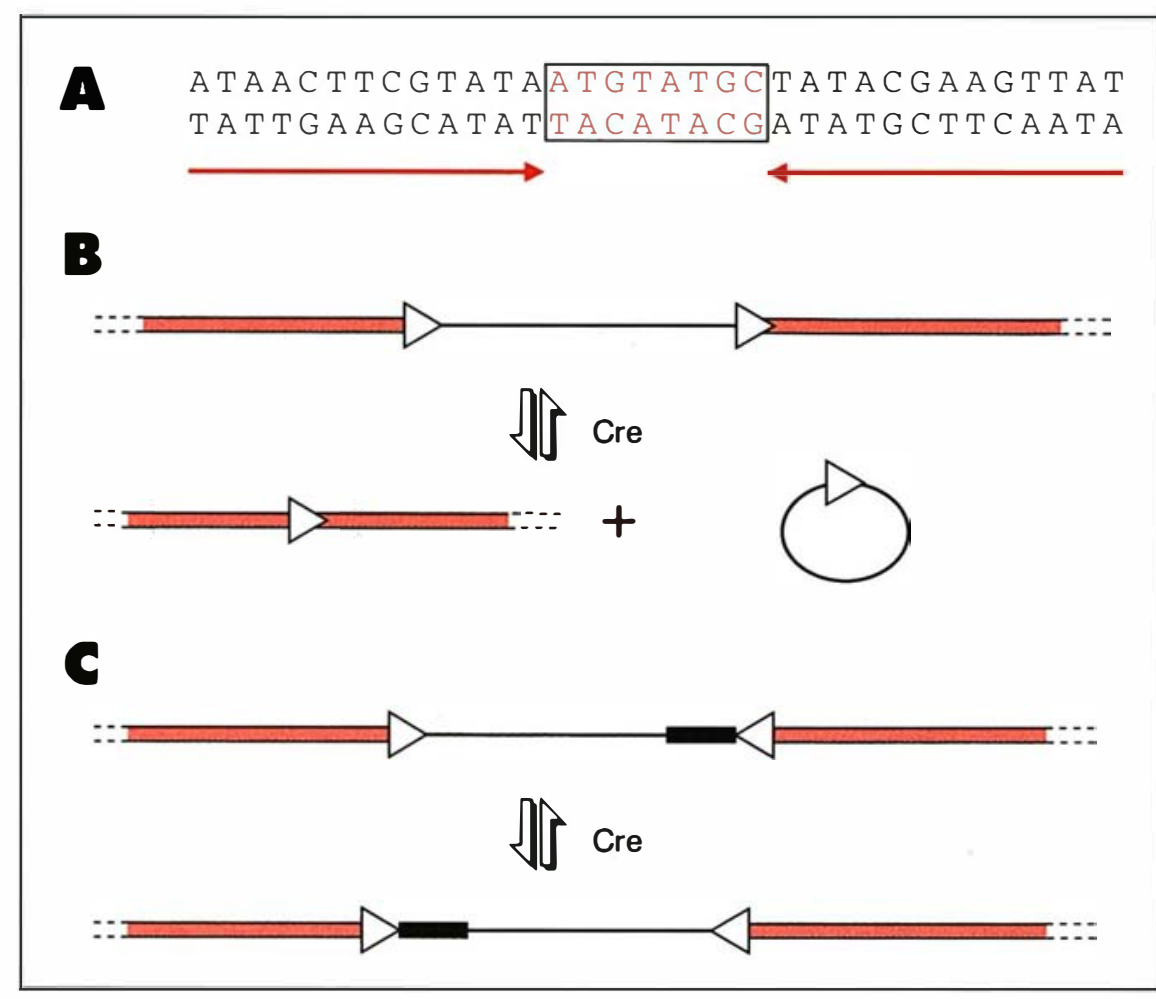

Figure 1. Le site LoxP et l'activité de I'enzyme Cre. A. Le site LoxP est composé de deux séquences palindromiques de $13 \mathrm{pb}$, soulignées par des flèches, séparées par une séquence asymétrique de $8 \mathrm{pb}$ (encadrées). B. Si deux sites LoxP (représentés par des triangles ouverts) sont placés dans la même orientation, le traitement par l'enzyme Cre conduit à la délétion du fragment $d^{\prime} A D N$ situé entre les deux sites, sous une forme circulaire qui sera rapidement dégradée. C. Si les deux sites LoxP sont dans des orientations opposées, le traitement par l'enzyme Cre conduit à l'inversion du fragment $d^{\prime} A D N$ situé entre les deux sites. Dans tous les cas, cette réaction est réversible car indépendante de l'énergie. (Figure tirée de l'article de $S$. Viville, médecine/sciences $n^{\circ} 5$, vol. 11, p. 735-46).

Figure 2 A. Principe de la stratégie employée pour obtenir une translocation réciproque entre les chromosomes 12 et 15 de souris. Les marqueurs de sélection sont introduits successivement par recombinaison homologue sur les chromosomes 12 et 15 en même temps qu'un "demiminigène " HPRT $5^{\prime}$ (chromosome 12) ou 3' (chromosome 15) et un site LoxP, orienté convenablement. Seule une recombinaison au site LoxP entre les chromosomes 12 et 15 permet de reconstituer un minigène HPRT complet entraînant la possibilité de croître en milieu de sélection HAT, les cellules devenant HPRT. Les cellules ES de départ sont HPRTet le restent après les deux événements de recombinaison homologue. La pointe de flèche blanche incluse dans chacun des rectangles indique le sens de la transcription $\left(5^{\prime} \rightarrow 3^{\prime}\right)$. néo: gène de résistance à la néomycine. hygro: gène de résistance à l'hygromycine. B. Minigène complet (1) et "demi-minigènes " 5" (2) et 3' (3) HPRT avec leurs sites LoxP. L'orientation de la partie centrale asymétrique des sites LoxP (voir figure 1) est indiquée. P: promoteur (actif dans les cellules ES). Rectangles bistres: exons codants. Rectangles hachurés: séquence de polyadénylation de SV4O.

Demi-minigène HPRT3 avec un site Lox $P$ 
Figure 3. Ciblages des gènes IgH sur le chromosome 12 (A) et c-myc sur le chromosome 15 (B), avec introduction d'un site LoxP et d'un " demi-minigène " HPRT (partie 5') dans le cas d'lgH et d'un "demiminigène " HPRT (partie $3^{\prime}$ ) dans le cas de c-myc. Les vecteurs utilisés sont des vecteurs de remplacement permettant une double sélection positive et négative $[2,13]$ avec dans le premier cas des cassettes tk et néo, dans le deuxième cas des cassettes tk et hygro. Les cellules ES recombinées homologues au locus IgH (cellules ES1) sont résistantes au ganciclovir (ganc ${ }^{R}$ ) du fait de la perte de la cassette tk, résistant à la néomycine $\left(n e ́ O^{R}\right)$, et restent HPRT-. Le deuxième événement de recombinaison homologue, effectué sur les celIules ES1, a lieu au locus c-myc et donne les cellules ES2 qui sont gan$\mathrm{c}^{\mathrm{R}}$, néo ${ }^{\mathrm{R}}$ (du fait du ciblage du gène $\mathrm{IgH}$ sur le chromosome 12), résistantes à l'hygromycine (hygro ${ }^{R}$ ) et restent HPRT-. Pour chacun des ciblages, les clones recombinés homologues sont identifiés par analyse en Southern blot; les sites de restriction $(H, X)$ et la taille des fragments attendus sont indiqués. Les sondes utilisées pour hybrider avec ces fragments sont représentées par des rectangles rouges hachurés. Lignes épaisses: séquences génomiques. Lignes fines : vecteur. $C$. Représentation détaillée de la translocation obtenue entre les chromosomes 12 et 15. Après expression de la recombinase Cre dans les cellules ES2, la translocation entraîne la reconstitution d'un minigène complet HPRT: en conséquence, seules les cellules porteuses de cette translocation pourront pousser en milieu HAT. La configuration chromosomique, au niveau de la translocation, des cellules ainsi sélectionnées (cellules ES3) a été vérifiée par analyse des fragments de restriction attendus en Southern blot (non montré). Rectangles rouges : exons ; $M$ : exon de la région membranaire $d^{\prime} \operatorname{lgH}$.

obtenir le réarrangement chromosomique recherché. Le moyen ingénieux utilisé par les auteurs a consisté à introduire avec chacun des sites LoxP un "demi-gène " de sélection

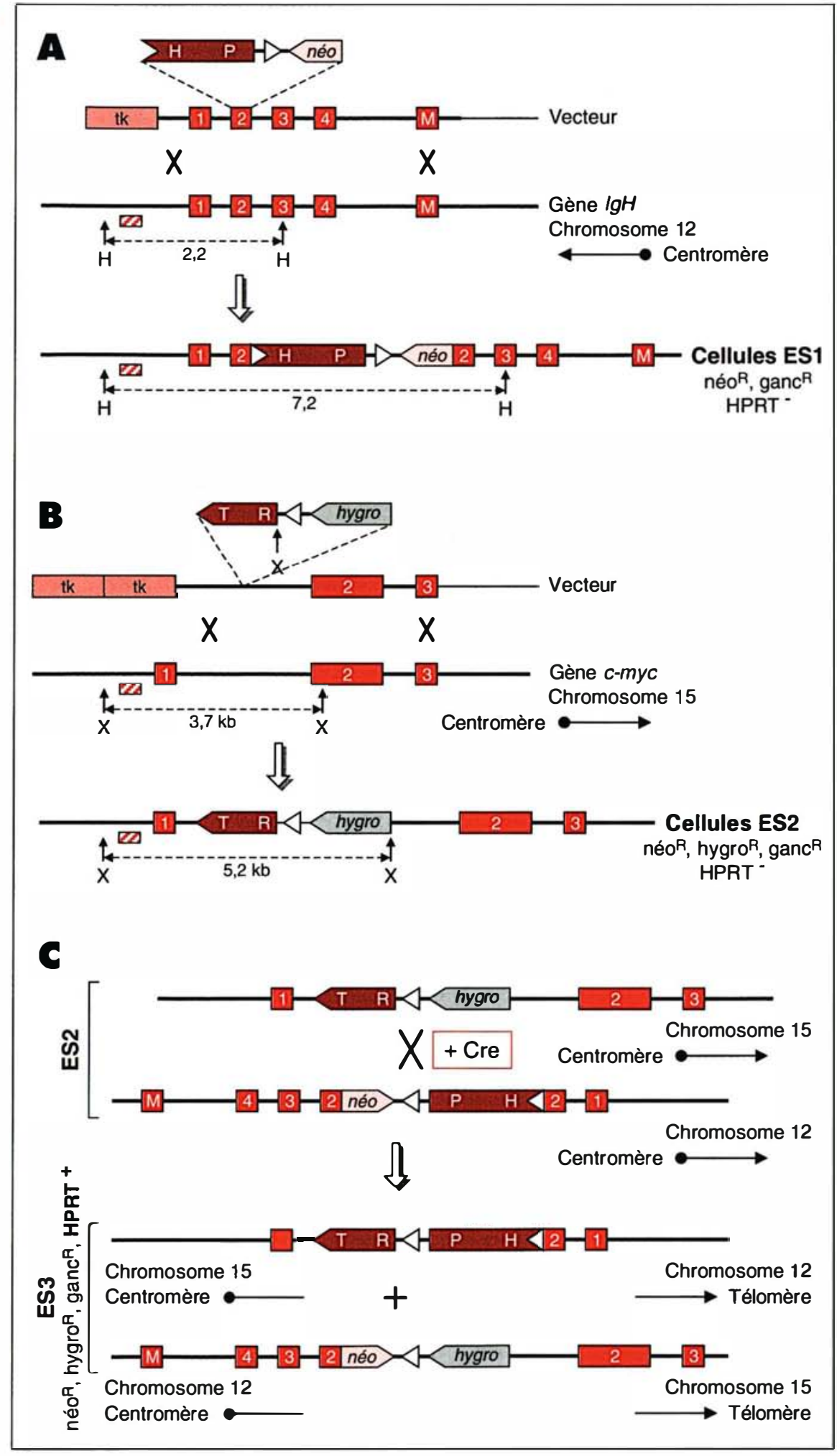

seules les cellules ayant subi le réarrangement chromosomique puissent pousser dans le milieu de sélection. Les étapes successives de l'obtention des cellules ES porteuses de la translocation ont été les suivantes: (1) obtention, à partir de cellules ES HPRT $^{-}$, de clones recombinants homologues au locus des chaînes lourdes d'immunoglobuline (chromosome 15) et porteurs d'un site LoxP, d'un gène de résistance à la 
néomycine et de la partie 5' d'un minigène HPRT. Ces recombinants homologues (cellules ES1) sont néo ${ }^{R}$, HPRT $^{-}$(figure $3 A$ ); (2) obtention à partir des cellules ESI de clones recombinés homologues au site $c-m y c$ et porteurs d'un deuxième site LoxP, d'un gène de résistance à l'hygromycine et de la partie 3' d'un minigène HPRT. Ces recombinants homologues (cellules ES2) sont néo ${ }^{R}$, hygro $^{\mathrm{R}}$, HPRT $^{-}$(figure 3B); (3) transfection dans les cellules ES2 d'un vecteur d'expression de la recombinase Cre, suivie d'une sélection des transformants HPRT ${ }^{+}$. Du fait de l'orientation relative des sites LoxP, par rapport aux centromères et aux minigènes 5' et 3' HPRT, on s'attend à ce que les clones $\mathrm{HPRT}^{+}$, sélectionnés en milieu HAT, soient porteurs d'une translocation réciproque entre les chromosomes 12 et 15 aux sites IgH et $c-m y c$ (figure $3 C$ ). En effet, seules les cellules ayant subi la recombinaison aboutissant à cette translocation devraient être capables de reconstituer un minigène HPRT complet et donc de pousser en milieu HAT (cellues ES3). Aussi bien l'analyse détaillée en Southern blot que l'utilisation de "coloration" de chromosomes par des sondes spécifiques a entièrement confirmé cette prédiction.

La translocation obtenue par Smith et al. reproduit un accident chromosomique observé dans les plasmocytomes, à deux différences près cependant. D'une part, la jonction des loci myc et $\operatorname{IgH}$ est interrompue par les cassettes de sélection, ce qui pourrait interférer avec l'activation du gène $c$ myc normalement observée dans les plasmocytomes. Pour pallier cet inconvénient, il suffirait en principe de modifier la position des cassettes de sélection néo ${ }^{R}$ et hygro ${ }^{R}$ par rapport aux séquences de sélection HPRT et aux sites LoxP, de manière à créer une jonction directe entre les séquences c-myc et $\operatorname{IgH}$ sur le chromosome transloqué. D'autre part, dans les souris éventuellement dérivées à partir des cellules ES3, le réarrangement obtenu serait constitutionnel (c'est-à-dire présent dans toutes les cellules) alors que, dans le cas des plasmocytomes et d'autres types de tumeurs [11], il n'est géné- ralement présent que dans les cellules tumorales. Il est, là aussi, théoriquement possible de tourner la difficulté en croisant des souris obtenues à partir des cellules ES2 (où la translocation n'a pas eu lieu) avec des souris transgéniques exprimant la recombinase sous la dépendance d'un promoteur spécifique du type cellulaire approprié (les lymphocytes B). Ainsi, dans les souris transgéniques issues de ce croisement, seul ce type cellulaire serait le siège de l'anomalie chromosomique. Un tel schéma expérimental s'est d'ailleurs avéré opérationnel dans un autre contexte [9] et pourrait être adapté à d'autres types de remaniements chromosomiques se produisant uniquement dans les cellules somatiques.

Un nouveau pas est donc franchi dans les possibilités de création programmée de modifications génétiques. Le travail de Smith et al. a démontré la possibilité d'obtenir des modifications à l'échelle chromosomique dans les cellules ES et ouvre donc la voie à la production de souris porteuses de ces modifications. En l'occurrence, il s'agit d'une translocation. Cependant, le même type de stratégie devrait permettre de créer également des inversions ou des délétions en des points précis des chromosomes qui, comme dans le cas des translocations, sont souvent associées à la formation de tumeurs ou à des anomalies du développement chez l'homme [11, 12]

\section{Charles Babinet}

Institut Pasteur, unité de biologie du développement, 25, rue du Docteur Roux, 75015 Paris, France.

\section{TIRÉS À PART}

C. Babinet.

\section{RÉFÉRENCES}

1. Lemarchandel V, Montagutelli X. La recombinaison homologue. De nouvelles perspectives pour la transgénèse chez les mammifères. médecine/sciences $1990 ; 6: 18$ 29.

2. Babinet C. Les cellules souches embryonnaires de souris: une voie privilégiée de transformation génétique à l'échelle de l'animal. médecine/sciences 1992 ; 8: 268-75.

3. Viville S. Recombinaison homologue: nouveaux vecteurs, nouvelles perspectives. médecine/sciences 1995; 11 : 735-46.

4. Capecchi MR. Altering the genome by homologous recombination. Science 1989 ; 244: 1288-92.

5. Koller BH, Smithies O. Altering genes in animals by gene targetting. Annu Rev Immunol 1992; 10: 705-30.

6. Kilby NJ, Snaith MR, Murray JAH. Sitespecific recombinase: tools for genome enginering. Trends Genet 1993; 9 : 413-21.

7. Zou YR, Müller W, Gu H, Rajewsky K. Cre-loxP-mediated gene replacement: mouse strain producing humanized antibodies. Curr Biol 1994; 4 : 1099-103.

8. Bronson SK, Smithies O. Altering mice by homologous recombination using embryonic stem cells. J Biol Chem 1994; 269 ; 271558.

9. Gu H, Marth JD, Orban PC, Mossmann $\mathrm{H}$, Rajewsky K Deletion of a DNA polymerase $\beta$ gene segment in $T$ cells using cellspecific gene targeting. Science $1994 ; 265$ : 103-6.

10. Smith AJH, De Sousa MA, Kwabi-Addo B, Heppell-Parton A, Impey H, Rabbitts P. A site-directed chromosomal translocation induced in embryonic stem cells by Cre-loxP recombination. Nature Genet 1995; 9 : 37685.

11. Rabbitts TH. Chromosomal translocations in human cancer. Nature $1994 ; 372$ 143-9.

12. Hall JG. How imprinting is relevant to human disease. Development 1990; Suppl. 141-8.

13. Mansour SL, Thomas KR, Capecchi MR Disruption of the proto-oncogene int-2 in mouse embryo-derived stem cells: a general strategy for targeting mutations to nonselectable genes. Nature 1988 ; 336: 348-52. 